\title{
Informática na educação especial: uma discussão no contexto da educação de surdos
}

\author{
Ronnie E. S. Santos ${ }^{1}$, Cleyton V. C. Magalhães ${ }^{1}$, Josinalva Maciel², Jorge S. \\ Correia-Neto $^{3}$, Guilherme Vilar ${ }^{3}$ \\ ${ }^{1}$ Centro de Informática (CIn) - Univ. Federal de Pernambuco (CIn/ UFPE) \\ Av. Jornalista Anibal Fernandes- Cid. Universitária, 50670-901 - Recife-PE - Brasil \\ ${ }^{2}$ Centro Educacional de Triunfo (CET Triunfo) \\ Triunfo - PE - Brasil \\ 3 Programa de Pós-Graduação em Informática Aplicada (PPGIA) - Universidade \\ Federal Rural de Pernambuco (UFRPE) \\ Rua Dom Manoel de Medeiros, s/n, Dois Irmãos, 52171-900 - Recife-PE - Brazil \\ \{ress, cvcm\}@cin.ufpe.br, jorgecorreianeto@gmail.com, \\ guilherme_vilardyahoo.com.br
}

Abstract. Context: In the context of education of students with special needs, specifically in education of deaf students, research in Informatics and Computer Science focuses on the development of tools, enhancing the adaptation of this community. Goal: The goal of this research is twofold: a) to perform a mapping study in the editions of RBIE (1997-2013), in the 13 last editions of SBIE (2001-2013) and in 10 editions of WIE (2003, 2005 - 2013) and; b) based on the results of the mapping study, to propose an educational software to deaf children. Method: The method used in this research is a mapping study, which is a secondary research method employed to integrate the results from previously published studies. Based on the results of the mapping study, a software to support deaf students to write texts in Portuguese was developed. Results: Results show that research related to education of deaf students were very discussed in the last decade in SBIE, WIE and RBIE. The main contribution of Brazilian community of Informatics in Education to the deaf community is the development of educational software and tools to support the teaching-learning process. Furthermore, based on the mapping study results, emerged the motivation to develop a basic software for text editing to support deaf students to create texts in Portuguese.

Resumo. Contexto: No contexto da educação especial, especificamente na educação de surdos, as pesquisas na área de computação e informática contribuem para o desenvolvimento de ferramentas, potencializando a adaptação desta comunidade. Objetivo: Os objetivos deste trabalho são: a) realizar um mapeamento sistemático nas edições da RBIE (1997 - 2013), nas 13 últimas edições do SBIE (2001 - 2013) e em 10 edições do WIE (2003, 2005 - 2013) e; b) com base nos resultados do mapeamento, propor um software educativo para alunos surdos. Método: $\mathrm{O}$ método utilizado nesta pesquisa é um mapeamento sistemático, método de pesquisa secundário empregado para integrar os resultados oriundos de diversos estudos publicados anteriormente. Com base nos resultados do mapeamento, foi desenvolvido um software para apoio a escrita de textos em português por estudantes surdos. Resultados: Os resultados mostram que pesquisas voltadas para a educação de surdos apareceu de forma constante na última década nos encontros do SBIE, WIE and RBIE. A principal contribuição da comunidade brasileira de Informática na Educação para a comunidade de surdos tem sido o desenvolvimento de software educativos e ferramentas de apoio ao processo de ensinoaprendizagem. Além disso, com base nos resultados do mapeamento, surgiu a motivação para desenvolver um ambiente de edição de textos com foco no auxílio a estudantes com deficiência auditiva na realização de atividades de elaboração de textos escolares em português. 


\section{Introdução}

Segundo dados do Censo Demográfico de 2010, mais 45 milhões de brasileiros afirmaram possuir algum tipo de deficiência visual, motora, auditiva ou mental. Deste total, cerca de 9,7 milhões de pessoas declaram ter deficiência auditiva, sendo que 344,2 mil pessoas são declaradas surdas (IBGE, 2012). Estes números têm justificado cada vez mais as ações para promover a inclusão da comunidade brasileira de surdos, especialmente em se tratando das questões de comunicação e acesso a educação.

$\mathrm{Na}$ realidade, por ser a linguagem dominante, a língua falada/oral quando apresentada na forma escrita acaba se tornando um grande desafio de comunicação para os surdos, sendo fundamental a disseminação das línguas de sinais. No Brasil, por exemplo, a língua brasileira de sinais (LIBRAS) é um sistema linguístico legítimo e natural, oficialmente utilizado pela comunidade surda brasileira. Essa modalidade gestual-visual tem uma estrutura gramatical independente da língua portuguesa falada no Brasil, pois, como nas diversas outras línguas de sinais, baseiam-se na identidade cultural dos surdos, que está ligada às experiências visuais (AZEREDO, 2006; QUADROS; PERLIN, 2007).

Os debates acerca da inclusão social dos surdos não se resumem apenas a diferenças de comunicação, mas também ao acesso desta comunidade a serviços essenciais, como a educação, por exemplo. Por esta e por outras questões, mais do que conhecer as necessidades da comunidade surda, pesquisadores das áreas de educação, assistência social, psicologia e informática, dentre outras, têm buscado meios pelos quais possam oferecer ferramentas de apoio adequadas e produzir conquistas importantes para a inclusão dos surdos na sociedade (TRINDADE; GARCIA, 2013).

No contexto geral da educação especial, especificamente na educação de surdos, as pesquisas na área de computação e informática contribuem para o desenvolvimento de ferramentas e avanços tecnológicos, potencializando a adaptação desta comunidade num mundo em que a maioria ouve. Desta forma, torna-se importante o desenvolvimento de estratégias educativas que melhorem a realidade de alunos surdos nas escolas (VIDAL; BARRETO, 2012). Neste contexto, considerando os três maiores meios de veiculação das pesquisas em informática na educação do Brasil, a Revista Brasileira de Informática na Educação (RBIE), o Simpósio Brasileiro de Informática na Educação (SBIE) e o Workshop de Informática na Escola (WIE), esta pesquisa possui dois objetivos principais:

a) Realizar um mapeamento sistemático nas edições da RBIE (1997 - 2013), nas 13 últimas edições do SBIE (2001 - 2013) e em 10 edições do WIE (2003, 2005 2013), para identificar e analisar as contribuições da comunidade brasileira de informática na educação para o processo de ensino-aprendizagem de alunos surdos;

b) Discutir as informações extraídas dos artigos analisados com uma especialista em educação de surdos e a partir desta discussão elaborar a proposta de um software educativo para alunos surdos, contribuindo assim para o crescimento da área.

O artigo segue organizado em cinco seções, iniciando nesta introdução. A segunda seção apresenta informações conceituais acerca do método científico adotado 
para a realização desta pesquisa, além de relatar experiências semelhantes no contexto da Informática na Educação. Na terceira seção são apresentadas as etapas metodológicas da pesquisa. Logo após, na quarta seção, os resultados do estudo são apresentados e discutidos e, por fim, a quinta seção apresenta as considerações finais.

\section{Estudos Secundários}

Estudos secundários são um tipo de pesquisa aplicada tipicamente na medicina e nas ciências sociais para integrar os resultados oriundos de diversos estudos primários, por exemplo, estudos de caso, experimentos, surveys, pesquisa-ação e etnografias (EASTERBROOK et al., 2011), que foram realizados para averiguar ou compreender fenômenos dentro de um contexto (KITCHENHAM; DYBÅ; JØRGENSEN, 2004). As revisões sistemáticas convencionais e mapeamentos sistemáticos são os dois tipos de estudos secundários mais comuns, utilizados em diversas outras áreas da computação.

As revisões sistemáticas convencionais agregam resultados sobre um problema específico e são aplicadas para resolver questões de pesquisa do tipo relacional e comparativa. Por outro lado, os mapeamentos sistemáticos são um tipo particular de revisão sistemática com uma visão mais ampla dos estudos primários e que busca responder questões descritivas sobre tópicos específicos (da SILVA et al., 2011). A realização de estudos secundários permite obter e avaliar um conjunto de evidências pertencentes a um contexto específico, com a finalidade de integrar resultados experimentais, e sua aplicação poderá enfatizar a descoberta de problemas gerais, além de incentivar e direcionar a elaboração de novas pesquisas naquele contexto (KITCHENHAM; DYBÅ; JØRGENSEN, 2004).

Considerando o contexto da Informática na Educação, recentemente Freitas Jr et al. (2012) analisaram as publicações internacionais sobre métodos de engenharia do conhecimento como suporte aos processos de aprendizagem organizacional e Aureliano e Tedesco (2012) investigaram nas publicações do SBIE e do WIE processos de ensinoaprendizagem de programação para iniciantes. Em 2013, Magalhães et al. discutiram e caracterizaram a pesquisa em Informática na Educação no Brasil com base nas publicações do SBIE e Araújo et al. (2013) mapearam o SBIE e o WIE e levantaram questões referentes ao desenvolvimento de software educacional inclusivo. $\mathrm{O}$ mapeamento sistemático proposto neste trabalho permitirá avaliar a contribuição da comunidade brasileira de Informática na Educação para o avanço no uso do computador no ensino-aprendizagem de alunos surdos, assim como identificar questões $\mathrm{e}$ necessidades de estudos futuros.

\section{Procedimentos Metodológicos}

Para realizar esse estudo, o processo de mapeamento sistemático foi operacionalizado em quatro etapas principais, baseado no processo para mapeamento sistemático em engenharia de software aplicado por Petersen et al. (2008).

\section{Etapa 1 - Definição do Escopo do Mapeamento}

Todo o planejamento do processo, definição das questões de pesquisa, critérios de busca e composição da equipe foi realizado nesta etapa, com a seguinte equipe: 
a) Dois estudantes de mestrado em Ciência da Computação, responsáveis por identificar, analisar e classificar os trabalhos publicados na revista e nos dois congressos, e relacionados com a questão de pesquisa;

b) Dois professores universitários, com experiência em pesquisas na área para discutir os resultados e resolver quaisquer divergências de classificação das publicações, nas as quais não houvesse um consenso entre os mestrandos;

Juntos os quatro pesquisadores realizaram a análise e classificação dos artigos para responder as questões divididas em duas facetas:

Aspectos Históricos: Como se deu a evolução histórica das pesquisas em informática na educação especial, especificamente no contexto de alunos surdos, publicadas pela comunidade brasileira de Informática na Educação?

Aspectos Conceituais: Quais as principais contribuições da comunidade brasileira de informática na educação para a comunidade de surdos?

\section{Etapa 2 - Busca e Seleção de Estudos Primários}

A busca ocorreu de forma manual através site da Comissão Especial de Informática na Educação da $\mathrm{SBC}$, de onde foram extraídos os artigos com disponíveis online, publicados na RBIE e também no SBIE e WIE. A figura 1 ilustra o processo de busca por estudos, a lista completa de todos os artigos analisados não pode ser incluída neste artigo por questões de espaço.

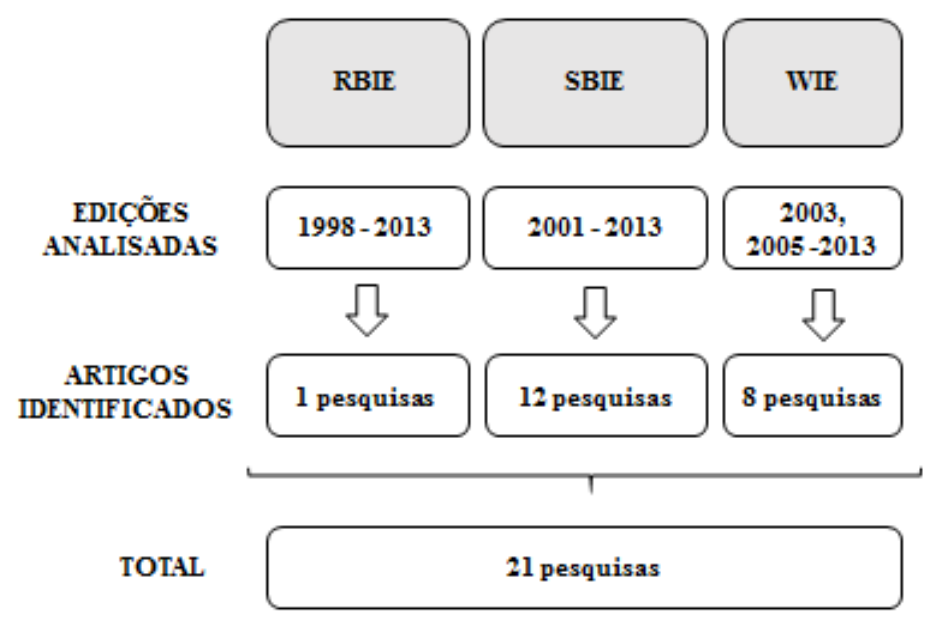

Figura 1. Processo de busca e seleção de estudos.

\section{Etapa 3 - Extração de dados}

Nesta fase buscou-se obter informações de cada um dos artigos encontrados, de forma a responder as perguntas de pesquisa. Para auxiliar este processo, um formulário de extração (Tabela 1) foi elaborado utilizando o MS Excel.

Tabela 1. Dados extraídos dos artigos selecionados.

\begin{tabular}{ll}
\hline \multicolumn{1}{c}{ Informação } & \multicolumn{1}{c}{ Descrição } \\
\hline Título do trabalho & Título do artigo publicado \\
\hline Meio de publicação & RBIE, SBIE ou WIE? \\
\hline Ano de publicação & Ano no qual o artigo foi publicado \\
\hline Instituição & Instituição à qual os autores dos artigos estão filiados \\
\hline
\end{tabular}




\begin{tabular}{ll}
\hline Problema de pesquisa & Qual o objetivo, motivação e problema de pesquisa do trabalho? \\
\hline Proposta & Solução computacional proposta pelos pesquisadores \\
\hline Público alvo & $\begin{array}{l}\text { Para que público a proposta é voltada? (professor, aluno de ensino } \\
\text { médio, graduandos, etc.). }\end{array}$ \\
\hline Tipo de proposta & $\begin{array}{l}\text { A proposta pode ser classificada como tutorial, exercício-e-prática, } \\
\text { programação, aplicativo, simulador, jogo educacional ou internet e } \\
\text { multimídia (VALENTE, 1999) }\end{array}$ \\
\hline Método empírico & Método empírico usado para validar os resultados da proposta \\
\hline Percepções & Principais contribuições da proposta \\
\hline
\end{tabular}

\section{Etapa 4 - Análise e Síntese}

A partir dos dados extraídos na fase anterior deu-se o processo de interpretação dos resultados, criação de tabelas e gráficos para apresentação da informação e descrição das evidências identificadas nos estudos primários. Além disto, os resultados desta análise foram apresentados para uma intérprete de LIBRAS, professora de uma escola da rede pública e com vasta experiência na educação de surdos. Com esta discussão foi possível propor uma ferramenta com uma abordagem diferente das identificas no mapeamento sistemático.

\section{Resultados e Discussões}

Esta seção tem o intuito de apresentar uma análise descritiva sobre as propostas que apoiam a educação especial com foco na comunidade de alunos surdos, publicadas pela comunidade brasileira de Informática na Educação nos últimos anos.

\subsection{Resultados do Mapeamento}

Aspecto Histórico: Como se deu a evolução histórica das pesquisas voltadas para a educação especial, especificamente tratando da comunidade de alunos surdos, pela comunidade brasileira de Informática na Educação?

A busca por pesquisas para a realização deste mapeamento sistemático compreendeu o período de 1998 a 2013, sendo que os primeiros trabalhos publicados com a temática da educação especial de surdos ocorreram em 2001 no SBIE. Foi identificada apenas uma proposta deste tipo na revista RBIE. Porém, pode-se observar que este tipo de pesquisa apareceu de forma constante na última década nos encontros do SBIE e WIE. Ao se considerar os números absolutos de publicações, pode-se dizer que ainda há pouco interesse da comunidade de Informática na Educação para esta temática, visto que 21 publicações é um número extremamente pequeno se considerarmos os mais de mil trabalhos publicados nos três meios no mesmo período.

A figura 2 mostra a distribuição das pesquisas por evento durante a última década. O SBIE foi o evento com mais contribuições (doze pesquisas), seguido do WIE com oito pesquisas sobre o tema. Ainda observando estes dados, verificou-se que em 2008, 2010 e 2012 nenhuma pesquisa com este foco foi publicada por estes meios, o mesmo não pode ser dito de 2002, pois mesmo não identificando nenhuma pesquisa na RBIE e no SBIE, a lista de trabalhos publicados no WIE não foi analisada neste ano, por não estar disponível na página da Comissão Especial de Informática na Educação. 


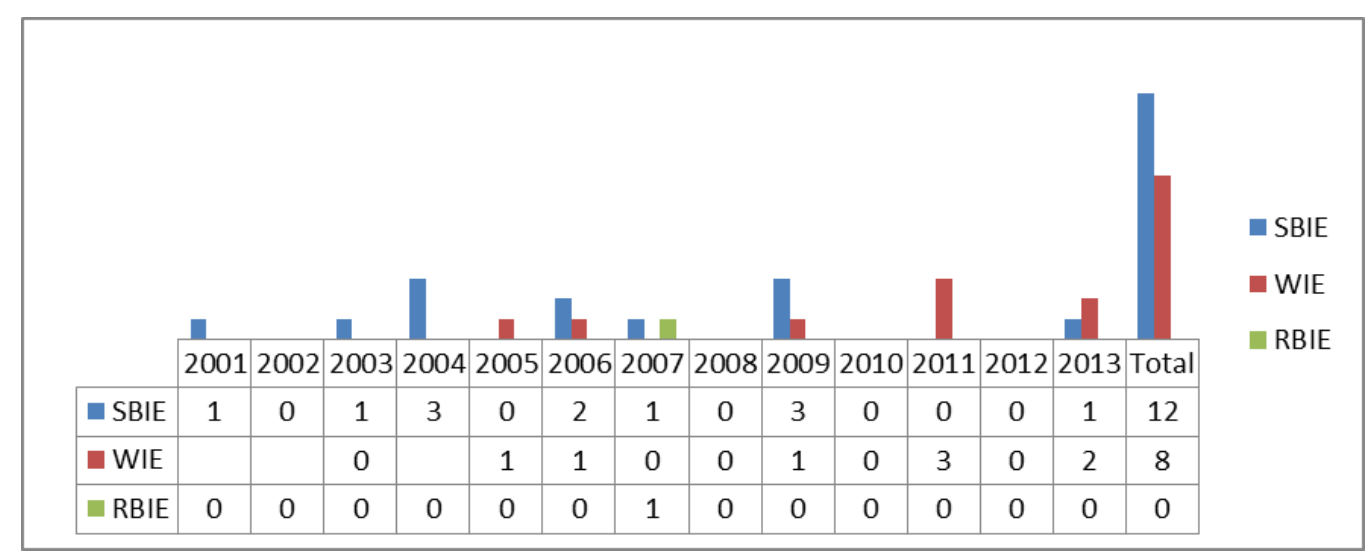

Figura 2. Distribuição das pesquisas voltadas para a educação especial de alunos surdos

Outra informação acerca da distribuição histórica das pesquisas que pode ser extraída foram as instituições que publicaram trabalhos com esta temática durante o período. Participaram deste processo autores de 20 instituições que contribuíram com pelo menos uma publicação, como mostra a tabela 2 .

Tabela 2. Instituições que publicaram pesquisa no contexto

\begin{tabular}{cl}
\hline Número de Publicações & \multicolumn{1}{c}{ Instituição } \\
\hline 3 Publicações & UFRGS, UNISINOS \\
\hline 2 Publicações & UFAM, UFRPE \\
\hline \multirow{2}{*}{1 Publicação } & UNIFRA, Universidade Metodista de Piracicaba, ULBRA, \\
& Universidade de Coimbra, FACENSA, UFPR, UENP, \\
& UNIVALI, UFMS, UNIPLAC, UCB, UFAL, UnB, \\
& FACOMP, UFRJ, UFC \\
\hline
\end{tabular}

A última informação histórica mapeada por esta pesquisa foi a relação entre os estudos identificados e o público alvo para o qual a ferramenta esteve direcionada. Neste aspecto, $76 \%$ dos estudos (16/21) tiveram foco principal na comunidade de estudantes surdos de forma geral, 19\% (4/21) foram criados para apoiar atividades com crianças em fase de alfabetização e 5\% (1/21) foi direcionado para alunos surdos em nível de graduação, mais especificamente graduação em Computação.

Aspectos Conceituais: Quais as principais contribuições da comunidade brasileira de informática na educação para a comunidade de surdos?

Pode-se se observar que a principal contribuição da comunidade brasileira de Informática na Educação para a comunidade de surdos tem sido o desenvolvimento de produtos de software educativos e ferramentas de apoio ao ensino-aprendizagem. Do total de estudos analisados, 90\% deles (19/21) apresentaram propostas inéditas de ambientes computacionais voltadas para este contexto, como por exemplo, sistemas de aprendizado de LIBRAS, sistemas de apoio à comunicação e sistemas de apoio ao professor mediador no processo de ensino (sendo um destes voltado para alunos surdos dos cursos de graduação em Computação). Além disto, duas propostas não apresentaram nenhum tipo de software: uma discutiu estratégias pedagógicas com o uso do computador no processo de ensino aprendizagem e a outra analisou a percepção do aluno surdo e de seus professores em relação ao uso de recursos de tecnologia da informação. 
Considerando a definição Valente (1999) para tipos de software educativo, podese classificar as propostas de ferramentas identificadas em quatro tipos: a) exercício e prática é a categoria com mais propostas, totalizando $58 \%(11 / 18)$ das pesquisas voltadas para o desenvolvimento de novas ferramentas; b) em segundo lugar as ferramentas do tipo internet e multimídia somam $21 \%$ das propostas de software educativo (4/19); c) por fim, com 11\% cada, aparecem as propostas de jogos (2/19) e simuladores (2/19). Percebe-se que os software do tipo exercício e prática são bastante comuns, pois com deles é possível implementar atividades centradas na ação e na memorização de informações.

Por fim, quando observado o método usado pelos pesquisadores percebeu-se que apenas cinco estudos utilizaram um método empírico para avaliar os resultados do estudo. Foram identificados dois estudos de caso, uma etnografia, um quasiexperimento e uma pesquisa-ação. As demais pesquisas apresentaram relatos de experiência e aplicação ou nenhum tipo de método avaliativo, apenas a descrição do sistema proposto. Pode-se concluir que esta temática tem apresentado um caráter pouco avaliativo ao longo dos anos e que o impacto das ferramentas propostas precisa ser medido e analisado empiricamente.

\subsection{Proposta de ferramenta}

Ao se discutir os resultados deste mapeamento sistemático com uma intérprete de LIBRAS, professora de alunos surdos em uma escola da rede pública, reforça-se a importância de propostas de software educativo para o contexto da educação de surdos. Porém, a especialista levanta uma questão importante: muitas ferramentas produzidas são descritas como auxiliares do processo de ensino aprendizagem de estudantes surdos, porém o seu desenvolvimento tem foco no português.

Segundo a especialista, a grande maioria das propostas identificadas seguem os princípios dos dicionários de LIBRAS online, nos quais para que o aluno surdo tenha acesso é necessário ter prévio conhecimento do português. Entretanto a língua materna destes alunos deveria ser a LIBRAS. Ainda segundo a intérprete, atualmente os alunos surdos de escola pública apresentam certo grau de dificuldade na aprendizagem do português, principalmente em se tratando de ortografia, pelo fato de muitas ferramentas terem foco no português (tradução de português para LIBRAS) e não na Língua de Sinais (tradução de LIBRAS para português), o que acaba não apoiando completamente estes alunos.

Diante desta dificuldade com ortografia, surgiu a motivação para desenvolver um ambiente de edição de textos com foco no auxílio a estudantes com deficiência auditiva e na realização de atividades de elaboração de textos escolares, visando a diminuição de erros ortográficos e de escrita. Com isso, além dos dados do mapeamento, esta pesquisa pode apresentar os resultados iniciais desta ferramenta cuja implementação seguiu a execução de quatro etapas:

- Etapa 1 - Seleção de sinais: a intérprete de LIBRAS identificou e enumerou os sinais mais utilizados no cotidiano de um deficiente auditivo;

- Etapa 2 - Categorização dos sinais: os sinais selecionados foram posteriormente divididos em categorias. Por exemplo, a categoria Família inclui palavras como pai, mãe, irmão, irmão, tia, tio, dentre outras. Foram definidas 15 categorias, 
cada uma contendo uma média de 20 sinais, escolhidos pela frequência de uso, segundo a intérprete;

- Etapa 3 - gravação dos sinais em vídeo: alunos surdos do ensino fundamental e médio de uma escola pública atuaram como intérpretes virtuais voluntários na gravação dos sinais que foram adicionados ao sistema;

- Etapa 4-Preparação das imagens: os vídeos foram convertidos para o formato gif e adicionados ao sistema com o seu respectivo termo em português. Desta forma, ao redigir um texto, o deficiente auditivo podia navegar pelas categorias e encontrar o respectivo termo em português.

A proposta inicial de editor de texto recebeu o nome de Deafword e carrega, na forma de sinais, o alfabeto e as primeiras categorias de palavras implementadas para a construção de textos referentes aos contextos família, apresentação pessoal, informática e tempo. A figura 2 apresenta a visão geral do sistema. A ideia é que o Deafword possa evoluir para uma ferramenta de autoria, na qual o próprio professor mediador possa gravar e adicionar na base de dados as suas expressões idiomáticas em LIBRAS, juntamente com o respectivo termo em português.

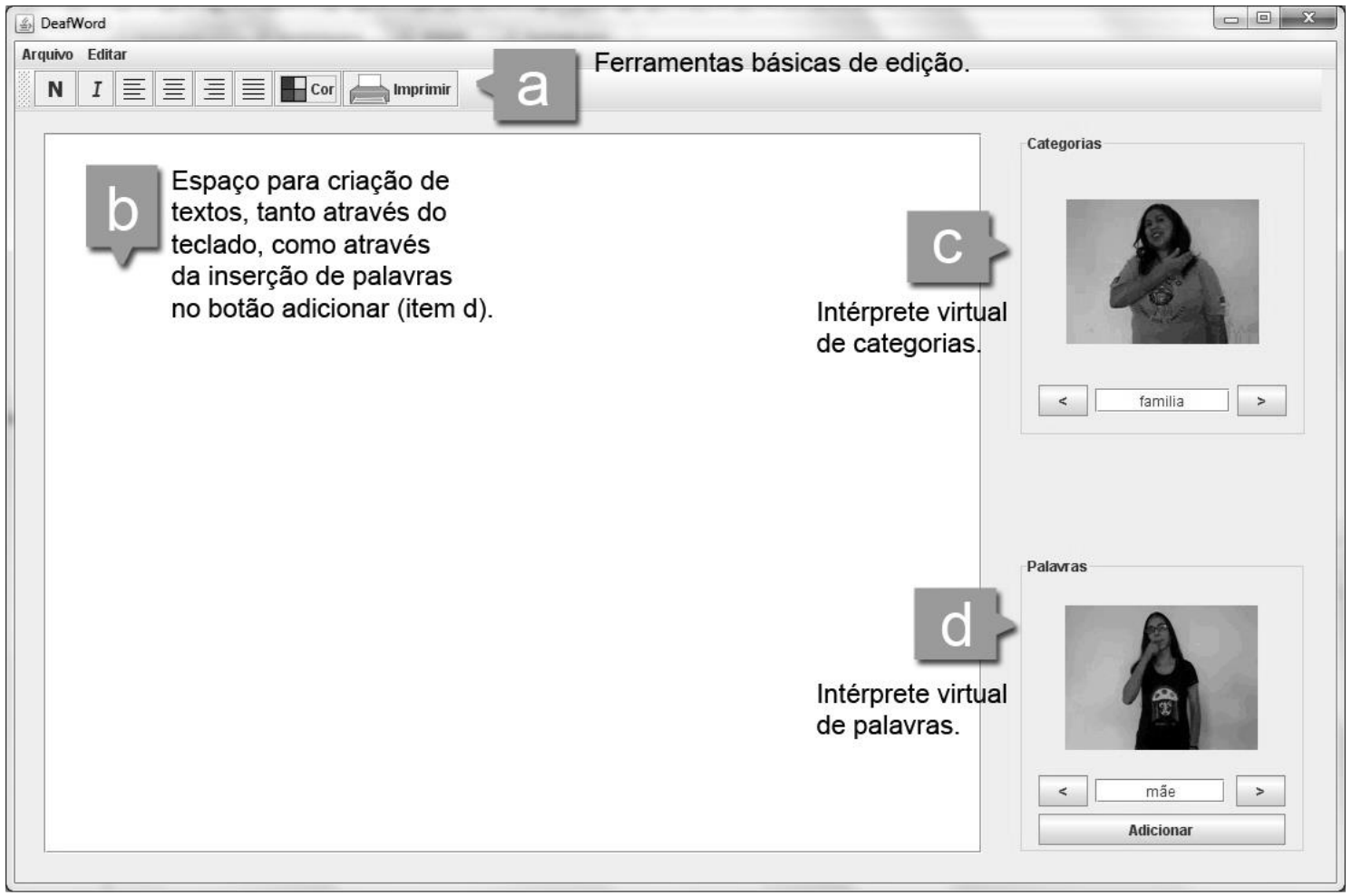

Figura 2. Deafword visão geral.

A primeira versão do Deafword para desktop permite desenvolver atividades de produção de texto com o auxílio de um intérprete virtual, que tem a função de diminuir a incidência de erros na escrita das palavras em português, uma vez que estas são apresentadas em diversas categorias na forma de LIBRAS. No lado direito da tela encontram-se os dois intérpretes virtuais representando as categorias (item c) e os sinais (item d) em LIBRAS. Desta forma, o aluno poderá navegar entre as categorias e adicionar o termo ao seu texto com a grafia correta em português, reduzindo assim o 
número de erros ao redigir a palavras. Por exemplo, caso o aluno não saiba a grafia correta do termo esposa, basta navegar pelas categorias (pelos botões avançar " $>$ " e retroceder "<") até encontrar, através do sinal, a categoria família e em seguida, buscar a palavra desejada (neste caso esposa), entre os demais sinais da categoria e adicionar a palavra corretamente ao texto. A área em branco (item b) à esquerda trata-se do local reservado para criação e edição dos textos.

Nesta primeira versão, o sistema apresenta as funções básicas de edição de textos, formatação de fonte, alinhamento, cores e também as rotinas de impressão (item a) e alinhamento de textos. Além disso, através do menu Editar é possível utilizar as funções de copiar e colar, comuns e sistemas deste tipo. É importante destacar que as imagens dos intérpretes não são estáticas, ou seja, ao utilizar a ferramenta o deficiente auditivo visualiza o sinal por completo, o que deixa o sistema mais eficiente para aqueles que ainda têm dificuldades inclusive com a LIBRAS. A ferramenta está atualmente em fase de teste e de validação com um grupo de estudantes surdos, através do desenvolvimento de atividades de ditado e escrita.

\section{Conclusões}

A proposta inicial deste mapeamento sistemático foi o de descrever quais as contribuições das pesquisas brasileiras em Informática na Educação no contexto do ensino-aprendizagem de alunos surdos. Pode-se dizer que ao longo do período analisado a comunidade brasileira de Informática na Educação apresentou propostas diversificadas sobre esta temática, entretanto, o número absoluto de pesquisas ainda é bastante reduzido.

Ainda existem muitas lacunas a serem resolvidas, principalmente em se tratando de ferramentas com foco principal no aprendiz e na língua de sinais, como apontado pela especialista na área. Neste contexto, a ideia inicial do editor de textos com foco na LIBRAS permite a elaboração de diversas atividades de apoio a escrita e ortografia da língua portuguesa para alunos surdos. Mas sabe-se que nesta proposta inicial uma das principais limitações é o armazenamento do material utilizado para representação dos termos em LIBRAS. Ainda é preciso otimizar o armazenamento e a busca pelas imagens, principalmente com a utilização de soluções na web ao invés de um banco de dados local para a realização de buscas mais dinâmicas e algoritmos de inteligência artificial, como os de reconhecimento de imagem. Outra alternativa para a otimização do banco de imagens seria a criação de um banco colaborativo, no qual as categorias e palavras relacionadas a cada categoria poderiam ser adicionadas por intérpretes cadastrados.

Outra proposta que também merece atenção é a realização de estudos de carácter avaliativo: de avaliação de aprendizagem de estudantes surdos suportado por computador, usabilidade de produtos de software educativos voltados para este tipo de aprendiz e relatos de experiência do uso de computador no ensino de alunos surdos. Também não foram identificadas experiências com este tipo de público no contexto das redes sociais e pouco se sabe sobre o acesso de estudantes surdos aos cursos superiores de graduação em Computação.

Os trabalhos futuros desta pesquisa incluem desenvolver novas funcionalidades de edição de textos para a melhoria do editor proposto, bem como a inclusão de novos 
termos e categorias de palavras. Estudam-se também formas de integrar a ferramenta com ambientes de redes sociais como o Twitter, para que os estudantes com deficiência auditiva possam realizar postagens diretamente com o auxílio da ferramenta. Também está prevista a realização de atividades e oficinas para validação desta primeira versão da ferramenta com estudantes surdos e com outros profissionais especialistas no assunto.

\section{Referências}

AURELIANO, Viviane Cristina Oliveira; TEDESCO, Patrícia Cabral de Azevedo Restelli. Ensino-aprendizagem de Programação para Iniciantes: uma Revisão Sistemática da Literatura focada no SBIE e WIE. Anais do $23^{\circ}$ Simpósio Brasileiro de Informática na Educação (SBIE 2012), ISSN 2316-6533. Rio de Janeiro, 2012.

AZEREDO, Eduardo. Língua Brasileira De Sinais "Uma Conquista Histórica". Brasília, 2006. Disponível em $<$ http://www.culturasorda.eu/resources/Reconocimiento_LIBRAS.pdf>. Acesso em: 18 abr. 2012.

da SILVA, F. Q. B.; SANTOS, A. L. M.; SOARES, S. C. B.; FRANÇA, A. C. C.; MONTEIRO, C. V. F.; MACIEL, F. F. Six years of systematic literature reviews in software engineering: An updated tertiary study. Information and Software Technology, 2011.

EASTERBROOK, S.; SINGER, J.; STOREY, M.; DAMIAN, D. Selecting Empirical Methods for Software Engineering Research. Computer Science Department, University of Toronto, 2011.

FREITAS JR, Vanderlei; ANDERLE, Daniel Fernando; SANTOS, Jane Lucia S.; STEIL, Andrea Valéria. Engenharia do Conhecimento como suporte aos processos de Aprendizagem Organizacional. Anais do $23^{\circ}$ Simpósio Brasileiro de Informática na Educação (SBIE 2012), ISSN 2316-6533. Rio de Janeiro, 2012.

IBGE - Instituto Brasileiro de Geografia e Estatística (2012). Censo demográfico 2010: Características gerais da população, religião e pessoas com deficiência. Rio de JaneiroRJ.

KITCHENHAM, B.; DYBÅ, T.; JØRGENSEN, M. Evidence-based Software Engineering. 26th International Conference on Software Engineering, (ICSE '04), Proceedings. IEEE, Washington DC, USA, pp 273 - 281, 2004.

MAGALHÃEES, Cleyton Vanut Cordeiro; SANTOS, Ronnie Edson de Souza; da SILVA, Fabio Queda Bueno; GOMES, Alex Sandro Caracterizando a Pesquisa em Informática na Educação no Brasil: Um Mapeamento Sistemático das Publicações do SBIE. Anais do 24ํㅗㅇ Simpósio Brasileiro de Informática na Educação (SBIE 2013). Campinas, 2013.

ARAÚJO, Ana Liz Souto Oliveira; BRITO, Rozimar Rodrigues; SILVA, Adriano Patrício Softwares para educação inclusiva: uma revisão sistemática no contexto de SBIE e WIE. Anais do $24^{\circ}$ Simpósio Brasileiro de Informática na Educação (SBIE 2013). Campinas, 2013.

QUADROS, Ronice Müller; PERLIN, Gladis. Estudos surdos II. Petrópolis, RJ: Arara Azul, 2007.

TRINDADE, Daniela de Freitas Guilhermino; GARCIA, Laura Sanchez. Framework Conceitual de apoio ao Design de Ambientes Colaborativos inclusivos aos Surdos. In XXIV Simpósio Brasileiro de Informática na Educação. Campinas, 2013.

VALENTE, José Armando. O computador na sociedade do conhecimento - Campinas, SP: Unicamp/NIED, 1999.

VIDAL, Livia Ferreira; BARRETO, Maria Auxiliadora Motta. Software Educacional: Uma Ferramenta Auxiliar na Alfabetização de Surdos. III Encontro Nacional de Ensino de Ciências da Saúde e do Ambiente. Niterói-RJ, 2012. 